\title{
INFINITELY DIFFERENTIABLE GENERALIZED LOGARITHMIC AND EXPONENTIAL FUNCTIONS
}

\author{
PETER WALKER
}

\begin{abstract}
We construct infinitely differentiable solutions of the functional equation $f(x+1)=e^{f(x)}$. Numerical values are found and their accuracy is discussed.
\end{abstract}

\section{INTRODUCTION}

Functions $F, G$ satisfying

$$
F(x+1)=e^{F(x)}
$$

and

$$
G\left(e^{x}\right)=G(x)+1
$$

are called generalized exponential and logarithmic functions, respectively. They are important in numerical analysis, where they are used in a new system of computer arithmetic which has significant advantages over floating-point arithmetic, including freedom from overflow and underflow, and a more satisfactory error measure. Details of this can be found in [1] and [2].

More generally, solutions of the Abelian functional equation

$$
f(x+1)=\phi(f(x)),
$$

where $\phi$ is a given function mapping a set $X$ to itself, are important in studying the flow on $X$ determined by $\phi$. This is because the inverse function $g$ (suitably defined) satisfies

$$
g(\phi(x))=g(x)+1,
$$

and the composition $\phi_{t}(x)=f(g(x)+t), t \in \mathbf{R}$, satisfies the formal identities $\phi_{0}(x)=x, \phi_{1}(x)=\phi(x)$, and $\phi_{t+u}(x)=\phi_{t}\left(\phi_{u}(x)\right)$.

In the case $X=\mathbf{C}, \phi(x)=e^{x}-1$, the author showed [7] that (3) has a nonconstant entire solution. Mapping properties of this function and its inverse in C can be found in [9]. Numerical values are calculated in [6], and by a different method in [8]. 
The case $X=\mathbf{C}, \phi(x)=e^{x}$ is of particular interest and difficulty owing to the absence of a real fixed point and the complicated nature of the trajectories of the exponential function, as is shown for instance in [3]. The existence of a real-analytic solution of (2) in this case was proved by Kneser [5], but that method did not allow numerical values to be calculated.

In this paper we show how to construct a $C^{\infty}$ solution of (2) by the use of a $C^{\infty}$ auxiliary function $h$ which satisfies

$$
h\left(e^{x}\right)=e^{h(x)}-1, \quad x \in \mathbf{R} .
$$

The numerical values of $h$ are easy to calculate since the iterative procedure by which it is defined is rapidly convergent. Section 2 gives the construction of $h$ and shows that it has the required properties. It is not clear whether $h$ can be continued analytically off the real axis: if this were possible, then we could relate our solution to the one found by Kneser.

This function $h$ is composed with the solution $g$ (which is known from [7] and [8]) of $g\left(e^{x}-1\right)=g(x)+1$, to give the required $G=g \circ h$. The major difficulty arises in the calculation of $g$ and this is discussed in $\S 3$.

Numerical values of $G$ are calculated in the range [0,1]; values outside this range can be found from the functional equation.

Other approaches to the problem which have been considered are discussed and compared in $\S 4$.

\section{THE AUXILIARY FUNCTION}

The required properties of $h$ are stated in Lemmas 1(iii), 2 and Theorem 1. These results may be taken on trust by a reader who wants to proceed directly to $\S 3$. We begin with the definition.

Definition 1. For $x \in \mathbf{R}$, let

$$
h_{0}(x)=x, \quad h_{1}(x)=\log \left(1+e^{x}\right),
$$

and generally for $n \geq 1$,

$$
h_{n}(x)=\log \left(1+h_{n-1}\left(e^{x}\right)\right) .
$$

We shall use the notations $l(x)=\log (1+x), f^{[n]}$ for the $n$th iterate of $f$, and $x_{n}=\exp ^{[n]}(x)$. In this notation $h_{n}(x)=l^{[n]}\left(x_{n}\right)$.

Lemma 1. (i) Each $h_{n}$ is increasing and real-analytic,

(ii) for all $x$, and $n \geq 1, h_{n}(x)>h_{n-1}(x)$, and

(iii) the limit $h(x)=\lim _{n \rightarrow \infty} h_{n}(x)$ exists, is continuous and satisfies $x<$ $h(x)<x+e^{-x}$ for all $x \in \mathbf{R}$. 
Proof. (i) is evident since both $e^{x}$ and $l(x)=\log (1+x)$ are increasing and real-analytic. For (ii) we see that for all $n \geq 0$ we have

$$
\begin{aligned}
h_{n+1}(x) & =l^{[n]}\left(\log \left(1+x_{n+1}\right)\right) \\
& =l^{[n]}\left(x_{n}+\log \left(1+1 / x_{n+1}\right)\right) \\
& >l^{[n]}\left(x_{n}\right)=h_{n}(x),
\end{aligned}
$$

since $l^{[n]}$ is increasing, and the result follows.

For (iii) observe that for $x>0$,

$$
\begin{aligned}
l^{[n]^{\prime}}(x) & =\frac{1}{1+l^{[n-1]}(x)} l^{[n-1]^{\prime}}(x) \\
& =\frac{1}{1+l^{[n-1]}(x)} \cdots \frac{1}{1+l^{[1]}(x)} \frac{1}{1+x},
\end{aligned}
$$

which is a positive decreasing function, bounded above by $1 /(1+x)$.

But for all $n \geq 1$ we have $x_{n}>0$, and so from the mean value theorem applied to the above expression for $h_{n+1}(x)$ we obtain on putting $k_{n}=$ $\log \left(1+1 / x_{n+1}\right)$ that

$$
\begin{aligned}
h_{n+1}(x) & =l^{[n]}\left(x_{n}+k_{n}\right) \\
& =l^{[n]}\left(x_{n}\right)+k_{n}\left(l^{[n]}\right)^{\prime}\left(x_{n}+\theta k_{n}\right), \quad 0<\theta<1, \\
& <h_{n}(x)+\log \left(1+1 / x_{n+1}\right) /\left(1+x_{n}\right) .
\end{aligned}
$$

In particular, we have the weaker inequality

$$
h_{n+1}(x)<h_{n}(x)+1 / x_{n+1},
$$

and since the series $\sum 1 / x_{n}$ converges with extreme rapidity, we deduce the uniform convergence of the sequence $\left(h_{n}\right)$, the continuity of $h(x)$, and the estimate $h(x)=x+O\left(e^{-x}\right)$ as $x \rightarrow \infty$.

It remains to prove the sharper inequality stated in (iii). It is enough to do this for $x \geq 0$, since for $x<0, h(x)$ is increasing while $x+e^{-x}$ is decreasing. From (5) we have

$$
\begin{aligned}
h(x) & <x+\log \left(1+e^{-x}\right)+\sum_{1}^{\infty}\left\{\log \left(1+1 / x_{n+1}\right)\right\} /\left(1+x_{n}\right) \\
& <x+\log \left(1+e^{-x}\right)+\left\{\sum_{1}^{\infty} 1 / x_{n+1}\right\} /\left(1+e^{x}\right),
\end{aligned}
$$

which we want to be $<x+e^{-x}$.

Hence, putting $u=e^{x} \geq 1$, we want

$$
A=\sum_{1}^{\infty} 1 / e^{[n]}(u)<B=(1+u)\{1 / u-\log (1+1 / u)\} .
$$

We show this by proving that $A<1 /(2 u)<B$. 
Note that $e^{u} \geq e-1+u$ for $u \geq 1$, and so $e^{[2]}(u) \geq e^{e+u-1}$, and similarly $e^{[n]}(u) \geq e^{[n]}(1) \cdot e^{u-1}$ for all $n \geq 1$. Thus,

$$
A \leq e^{1-u} \sum_{1}^{\infty} 1 / e^{[n]}(1)<e^{1-u} / 2<1 /(2 u) .
$$

For the other half of the required inequality, we have

$$
\begin{aligned}
B & =(1+u)(1 / u-\log (1+1 / u)) \\
& =(1+u) \sum_{2}^{\infty}(-1)^{n} /\left(n u^{n}\right) \\
& =\left[1+2 \sum_{2}^{\infty}(-1)^{n} /\left\{n(n+1) u^{n}\right\}\right] /(2 u),
\end{aligned}
$$

and the result follows since the sum of the series is positive.

Lemma 2. For all $x \in \mathbf{R}, h\left(e^{x}\right)=e^{h(x)}-1$, and $h$ is the unique solution of this functional equation which has the additional property that $x<h(x)<$ $x+$ constant, for $x \geq 0$.

Proof. The functional equation is immediate since

$$
h_{n-1}\left(e^{x}\right)=\exp \left(h_{n}(x)\right)-1 .
$$

Let $g$ be any other solution with $x<g(x)<x+C$. Then

$$
g(x)=l^{[n]}\left(g\left(x_{n}\right)\right)>l^{[n]}\left(x_{n}\right)=h_{n}(x),
$$

so $g(x) \geq h(x)$.

Also

$$
\begin{aligned}
g(x) & <l^{[n]}\left(x_{n}+C\right) \\
& =l^{[n]}\left(x_{n}\right)+C\left(l^{[n]}\right)^{\prime}\left(x_{n}+\theta C\right), \quad 0<\theta<1 ;
\end{aligned}
$$

the second term tends to zero as in Lemma 1, and the proof is complete.

Note. The uniqueness result evidently holds under very much less restrictive conditions.

Lemma 3. (i) For all $x \in \mathbf{R}$ and $n \geq 1$, we have $0<h_{n}^{\prime}(x)<1$.

(ii) Both $h$ and all $h_{n}$ are convex.

(iii) $h$ is differentiable on $\mathbf{R}$, and

$$
h^{\prime}(x)=\exp \left\{\sum_{0}^{\infty}\left(x_{n}-h\left(x_{n}\right)\right)\right\} .
$$

Also, $0<h^{\prime}(x)<1$ and $1-h^{\prime}(x)=O\left(e^{-x}\right)$ as $x \rightarrow \infty$. 
Proof. (i) We have $0<h_{1}^{\prime}(x)<1$ by inspection, and for $n \geq 1$

$$
h_{n}^{\prime}(x)=h_{n-1}^{\prime}\left(e^{x}\right) \exp \left(x-h_{n}(x)\right),
$$

from which (i) follows by induction since $h_{n}(x)>x$.

(ii) The convexity of $h_{n}$ (and thus of $h$ also) follows from (i) and

$$
\begin{aligned}
h_{n}^{\prime \prime}(x)= & h_{n-1}^{\prime \prime}\left(e^{x}\right) \exp \left(2 x-h_{n}(x)\right) \\
& +h_{n-1}^{\prime}\left(e^{x}\right)\left(1-h_{n}^{\prime}(x)\right) \exp \left(x-h_{n}(x)\right) .
\end{aligned}
$$

(iii) Since $h$ is convex, there is an at most denumerable set $D_{1}$ such that $h^{\prime}(x)$ exists for all $x \in \mathbf{R} \backslash D_{1}$ : also $h^{\prime}$ must have a limit as $x \rightarrow \infty$ through $\mathbf{R} \backslash D_{1}$, and this limit must equal 1 to satisfy Lemma 1(iii). For some larger (but still denumerable) set $D_{2}$ we can suppose that $h^{\prime}(x)$ and $h^{\prime}\left(x_{n}\right)$ exist for all $x \in \mathbf{R} \backslash D_{2}, n \geq 1$. Then from the functional equation for $h$,

$$
h^{\prime}(x)=h^{\prime}\left(x_{n}\right) \exp \left\{\sum_{0}^{n}\left(x_{r}-h\left(x_{r}\right)\right)\right\},
$$

and so, letting $n \rightarrow \infty$,

$$
h^{\prime}(x)=\exp \left\{\sum_{0}^{\infty}\left(x_{r}-h\left(x_{r}\right)\right)\right\}
$$

for $x \in \mathbf{R} \backslash D_{2}$. But the summation is continuous, so $h$ must be continuously differentiable and this expression for $h^{\prime}(x)$ is proved for all $x \in \mathbf{R}$, and in addition $0<h^{\prime}(x)<1$. The last statement follows as in the proof of Lemma 1(iii).

Lemma 4. The estimate $0<x_{n}^{(k)} \leq(k-1) ! n^{k-1}\left(x_{n}\right)^{2 k}$ holds for all $n \geq 0$, $k \geq 1$. For $x \geq 0$, we have $x_{n} \geq n$, and the weaker but more useful consequence that

$$
x_{n}^{(k)} \leq(k-1) !\left(x_{n}\right)^{3 k-1}
$$

Proof. Since $x_{1}=e^{x}, x_{n}=\exp \left(x_{n-1}\right)$, we have.

$$
x_{n}^{\prime}=x_{n} x_{n-1}^{\prime}=x_{n} x_{n-1} \cdots x_{1} \text {. }
$$

But for all $x \geq 0$, both $x$ and $x^{2}$ are $<e^{x}$, so $x_{1} x_{2}<x_{2}^{2}, x_{1} x_{2} x_{3}<x_{2}^{2} x_{3}<$ $x_{3}^{2}$, and generally

$$
x_{n}^{\prime}=x_{1} \cdots x_{n}<x_{n-1}^{2} x_{n}<x_{n}^{2} .
$$

This gives the result for $k=1$.

Differentiate again to get

$$
x_{n}^{\prime \prime}=x_{n}^{\prime}\left(x_{n}^{\prime}+\cdots+x_{1}^{\prime}\right)=x_{n}^{\prime} \sigma_{n}^{\prime}
$$

say, where

$$
\sigma_{n}=x_{1}+\cdots+x_{n}
$$


and

$$
\sigma_{n}^{(k)}=x_{1}^{(k)}+\cdots+x_{n}^{(k)} \leq n x_{n}^{(k)} .
$$

Hence $x_{n}^{\prime \prime} \leq n x_{n}^{\prime 2}$, which is the result when $k=2$.

Suppose the result is proved for $r=1, \ldots, k$. Differentiate

$$
x_{n}^{\prime \prime}=x_{n}^{\prime} \sigma_{n}^{\prime}
$$

$(k-1)$ times to get

$$
\begin{aligned}
x_{n}^{(k+1)} & =\sum_{r=0}^{k-1}\left(\begin{array}{c}
k-1 \\
r
\end{array}\right) x_{n}^{(r+1)} \sigma_{n}^{(k-r)} \\
& \leq \sum_{r=0}^{k-1}\left(\begin{array}{c}
k-1 \\
r
\end{array}\right) r ! n^{r}\left(x_{n}\right)^{2(r+1)}(k-r-1) ! n^{k-r}\left(x_{n}\right)^{2(k-r)} \\
& =(k-1) !\left(x_{n}\right)^{2(k+1)} \sum_{r=0}^{k-1} n^{k}=k ! n^{k}\left(x_{n}\right)^{2(k+1)},
\end{aligned}
$$

as required.

Theorem 1. The function $h$ is $C^{\infty}$ on $\mathbf{R}$.

Proof. We show inductively that $h$ is $k$ times differentiable and that both $1-h^{\prime}(x)$, and $h^{(k)}(x)$ for $k \geq 2$, are $O\left(e^{-x}\right)$ as $n \rightarrow \infty$. Lemma 3(iii) gives the case $k=0$.

For the general case, let $S(x)=\sum_{0}^{\infty}\left(x_{n}-h\left(x_{n}\right)\right)$, so we already know that $S(x)=O\left(e^{-x}\right)$ as $x \rightarrow \infty$, and that $h^{\prime}(x)=e^{S(x)}$.

It follows that $S$ is differentiable with

$$
S^{\prime}(x)=\sum_{0}^{\infty}\left(1-h^{\prime}\left(x_{n}\right)\right) x_{n}^{\prime},
$$

where the series converges and its sum is $O\left(e^{-x}\right)$ by comparison with $\sum_{0}^{\infty} e^{-x_{n}}\left(x_{n}\right)^{2}$, using Lemma 4. Hence $h$ is twice differentiable, $h^{\prime \prime}(x)=$ $e^{S(x)} S^{\prime}(x)$, and this also is $O\left(e^{-x}\right)$. Now $S$ is twice differentiable and

$$
S^{\prime \prime}(x)=\sum_{0}^{\infty}\left\{\left[1-h^{\prime}\left(x_{n}\right)\right] x_{n}^{\prime \prime}-h^{\prime \prime}\left(x_{n}\right)\left(x_{n}^{\prime}\right)^{2}\right\},
$$

where the convergence of the series is assured by Lemma 4 .

We proceed inductively in this way. Having shown that $S$ is $k-1$ times differentiable and that $S^{(k-1)}(x)=O\left(e^{-x}\right)$, the existence of $h^{(k)}$ is deduced from $h^{\prime}=e^{S}$ by $k-1$ differentiations, each term being again $O\left(e^{-x}\right)$. Then from $S(x)=\sum_{0}^{\infty}\left(x_{n}-h\left(x_{n}\right)\right)$ we see by $k$ differentiations and a suitable majorization from Lemma 4 that $S^{(k)}$ exists and is $O\left(e^{-x}\right)$ as $n \rightarrow \infty$. This completes the proof of Theorem 1 . 


\section{VALUES OF GENERALIZED LOGARITHMS}

We construct a generalized logarithm as the composition $G=g \circ h$, where $h$ is the auxiliary function defined in $\S 2$, and $g$ is defined on $\Omega=\mathbf{C} \backslash(-\infty, 0]$ as follows.

Let $l(z)=\log (1+z)$ (the principal value on $\mathbf{C} \backslash(-\infty,-1])$, let $z_{0}=z$, and for $n \geq 1$, let $z_{n}=l\left(z_{n-1}\right): z_{n}=l^{[n]}(z)$ in the notation of $\S 2$. Then it can be proved (essentially as in [4]) that $z_{n} \rightarrow 0$ as $n \rightarrow \infty$, and that the limit

$$
g(z)=\lim _{n \rightarrow \infty}\left(n-(\log n) / 3-2 / z_{n}\right)
$$

exists locally uniformly on $\Omega$ and satisfies

$$
g(l(z))=g(z)-1, \quad z \in \Omega .
$$

The restriction of $g$ to $(0, \infty)$ is the function we require. The above limit is attained too slowly to be of use for numerical calculation; however, if we take further terms in the asymptotic expansion of $2 / z_{n}$ we obtain [8, Theorem 2 and Lemma 4]

$$
\begin{aligned}
2 / z_{n}= & n-W_{n} / 3+\left(W_{n}-1 / 2\right) / 9 n \\
& +\left(W_{n}^{2}-3 W_{n}+7 / 5\right) / 54 n^{2}+O\left(W_{n} / n\right)^{3},
\end{aligned}
$$

where $W_{n}=3 g(z)+\log n$.

Discarding the error term in (6) we obtain a quadratic equation for $W_{n}$ with one large spurious root and a small root which gives the required value of $W_{n}$ and so of $g$.

In Table 1 we give the value of $g(1)$ found in this way for successively larger values of $n$. The values were calculated in quadruple-precision arithmeticonly the most significant figures are shown.

For the accuracy we may argue heuristically as follows. Each successive row in the table corresponds roughly to a doubling of $n$, and gives one extra digit in agreement between successive calculated values: this is consistent with the $O(\log n / n)^{3}$ form of the error term in (6). This persists until around $n=10^{6}$, at which time accumulated errors from the iteration of $l(x)$ start to overwhelm all other contributions. This makes it reasonable to conjecture that the first fifteen or so leading figures in the last rows of Table 1 are correct.

For higher accuracy one could either take additional terms in the asymptotic expansion (6), or a more accurate routine for logarithms, though both of these possibilities leave unanswered the question of the actual precision of the calculated values of $g$ as defined by its iterative limit.

The same phenomenon is observed for other values of $x$, and values of $g(x)$ found in this way are given in Table 2.

The calculation of the auxiliary function $h$ presents no comparable difficulty. The estimate $0<h_{n+1}(x)-h_{n}(x)<1 / x_{n+1}$ established in Lemma 1 shows that $h_{N}(x)$ is already a sufficient approximation to $h(x)$, where $N$ is the least $n$ with $x_{n} \geq 174$ (when $x_{n+1} \geq 10^{75}$ ): for any real $x, N \leq 5$. 
TABLE 1

\begin{tabular}{|l|l|}
\hline$n$ & \multicolumn{1}{|c|}{ Value of $g(1): n$ terms } \\
\hline 10 & -2.256064 \\
20 & -2.256876 \\
50 & -2.256957939 \\
100 & -2.256960915 \\
200 & -2.256961142 \\
500 & -2.256961158561 \\
1000 & -2.256961158866260 \\
2000 & -2.256961158873057 \\
5000 & -2.256961158872499 \\
10000 & -2.256961158872501761 \\
20000 & -2.256961158872510526 \\
50000 & -2.256961158872512340 \\
100000 & -2.256961158872512361 \\
200000 & -2.256961158872512355 \\
500000 & -2.256961158872512353 \\
1000000 & -2.256961158872512576 \\
\hline
\end{tabular}

TABLE 2

\begin{tabular}{|r|r|}
\hline$x$ & $g(x)$ \\
\hline 1 & -2.25696115887251 \\
2 & -1.04845125695699 \\
3 & -0.59984484228986 \\
4 & -0.35509356624487 \\
5 & -0.19687054509995 \\
6 & -0.08424202242519 \\
7 & 0.00106837188459 \\
8 & 0.06854836014503 \\
9 & 0.12365559043878 \\
10 & 0.16977291895097 \\
\hline
\end{tabular}


TABLE 3

\begin{tabular}{|r|r|}
\hline$x$ & $\widehat{G}(x)$ \\
\hline-0.02 & -0.01820430135950 \\
-0.01 & -0.00912801055846 \\
0 & 0.00000000000000 \\
0.01 & 0.00917911671855 \\
0.02 & 0.01840869952666 \\
0.1 & 0.09397655687355 \\
0.2 & 0.19228332981427 \\
0.3 & 0.29391401137490 \\
0.4 & 0.39767124642776 \\
0.5 & 0.50226753353087 \\
0.6 & 0.60645065932094 \\
0.7 & 0.70911562706105 \\
0.8 & 0.80936431550622 \\
0.9 & 0.90650199352606 \\
1.0 & 1.00000000000000 \\
\hline
\end{tabular}

The values of the required generalized logarithm are then calculated as the composition $G=g \circ h$. Table 3 gives the normalized values $\widehat{G}(x)=G(x)-G(0)$ for $x=0(0.1) 1$. The extra values in the neighborhood of $x=0$ enable $G^{\prime}(0)$ to be estimated, for use in the final section where it is compared with the value found by other means.

\section{OTHER METHODS}

One easy way to construct a solution of equation (2) is to define $G(x)=x$ for $0 \leq x \leq 1$ and use the functional equation to extend the definition to the rest of $\mathbf{R}$. It is easy to verify that this gives a $C^{1}$ function which is not $C^{2}$.

To obtain a $C^{\infty}$ or even analytic solution, the author's "matrix method" [1], [8] assumes a power series for $G$ about $x=0$ in the form

$$
G(x)=\sum_{1}^{\infty} c_{n} x^{n}
$$

Substitute this into (2) and expand both sides to get the system of equations

$$
\sum_{1}^{\infty} c_{n}=1
$$

and

$$
\frac{1}{k !} \sum_{1}^{\infty} n^{k} c_{n}=c_{k}, \quad k \geq 1 \text {. }
$$


A possible way of solving these equations is to consider only the first $N$ terms of the first $N$ equations, thus obtaining $\left(c_{n}^{(N)}\right)$ as the solution of

$$
\sum_{1}^{N} c_{n}^{(N)}=1
$$

and

$$
\frac{1}{k !} \sum_{1}^{N} c_{n}^{(N)} n^{k}=c_{k}^{(N)}, \quad 1 \leq k \leq N-1 .
$$

The solutions found in this way appear initially to converge with increasing $N$ but for larger values of $N$ the numerical results are inconclusive and no proof of convergence is known. The values of $c_{1}^{(N)}$ found with $N=30, \ldots, 80$ are

$$
\begin{aligned}
& 0.9159437391 \ldots, N=30 \\
& 0.9159447811 \ldots, N=40 \\
& 0.9159452632 \ldots, N=50 \\
& 0.9159455362 \ldots, N=60 \\
& 0.9159457310 \ldots, N=70 \\
& 0.9159458315 \ldots, N=80 .
\end{aligned}
$$

By contrast, the value of $G^{\prime}(0)$ from Table 3 is 0.915356365 using a 3-point, and 0.91536681 using a 5-point formula. This discrepancy in only the fourth decimal place seems to indicate that the matrix method, if it converges at all, may converge to a different solution of (2).

The values of $G(x)$ found by the matrix method when $N=40$ are in Table 4 and show similar differences from those in Table 3.

TABLE 4

\begin{tabular}{|c|c|}
\hline$x$ & $G(x)$ \\
\hline 0 & 0.00000000000000 \\
0.1 & 0.09396824324443 \\
0.2 & 0.19213448275065 \\
0.3 & 0.29353262633353 \\
0.4 & 0.39705083840542 \\
0.5 & 0.50150089593100 \\
0.6 & 0.60569645133134 \\
0.7 & 0.70852774613322 \\
0.8 & 0.80902176748067 \\
0.9 & 0.90638109993332 \\
1.0 & 1.00000000000000 \\
\hline
\end{tabular}


These differences cannot be explained without at least a proof of convergence of the matrix method. And we cannot identify our function defined by the iteration method of $\S 3$, with Kneser's function defined by conformal mappings, without an extension of the domain of the function $h$ to include nonreal values. Until both these difficulties have been overcome, the possibility remains that either two or three distinct generalized logarithms have been constructed.

\section{ACKNOWLEDGMENTS}

The author thanks Professor F. W. J. Olver and Drs. J. M. Smith and D. W. Lozier (private communication) for the values of $c_{1}^{(N)}$ quoted in $\S 4$, and colleagues in Lancaster, Dhahran and Oman for helpful discussions and correspondence.

\section{BIBLIOGRAPHY}

1. C. W. Clenshaw, D. W. Lozier, F. W. J. Olver, and P. R. Turner, Generalized exponential and logarithmic functions, Comput. Math. Appl. 12B (5/6) (1986), 1091-1101.

2. C. W. Clenshaw, F. W. J. Olver, and P. R. Turner, Level-index arithmetic: an introductory survey, Numerical Analysis and Parallel Processing (P. R. Turner, ed.), Lecture Notes in Math., vol. 1397, Springer-Verlag, New York, 1989.

3. R. L. Devaney and M. Krych, Dynamics of $\exp z$, Ergodic Theory Dynamical Systems 4 (1984), 35-52.

4. P. Fatou, Sur les équations fonctionelles, Bull. Soc. Math. France 47 (1919), 161-271; 48 (1920), 33-94 and 208-314.

5. H. Kneser, Reelle analytische Lösungen der Gleichung $\phi(\phi(x))=e^{x}$ und verwandter Funktionalgleichungen, J. Reine Angew. Math. 187 (1948), 56-67.

6. K. W. Morris and G. Szekeres, Tables of the logarithm of iteration of $e^{x}-1$, J. Austral. Math. Soc. 2 (1961-1962), 321-333.

7. P. L. Walker, A class of functional equations which have entire solutions, Bull. Austral. Math. Soc. 39 (1988), 351-356.

8. $\ldots$ On the solutions of an Abelian functional equation, J. Math. Anal. Appl. 155 (1991) 93-110.

9.

College of Science, Sultan Qaboos University, P.O. Box 32486, Al-Khod, Muscat, Sultanate of OMan 